\title{
Effect of Nursing on Patients with Primary Liver Cancer before and after Transcatheter Arterial Embolization
}

\author{
LAN LUAN AND LIN WANG ${ }^{1 *}$
}

Department of Nursing, Jiangsu College of Nursing, Huai'an, 223005, 'Department of General Surgery, The Second People's Hospital of Huai'an (The Affiliated Huai'an Hospital of Xuzhou Medical University), Huai'an, 223002, China

\section{Luan and Wang: Nursing Effect for Patients with Primary Liver Cancer}

\begin{abstract}
The aim of the present study was to observe and analyse the nursing effect on primary liver cancer before and after transcatheter arterial embolization. About 200 patients with advanced primary liver cancer who had been treated in the Second People's Hospital of Huai'an were selected as research subjects. All of them were subjected to transcatheter arterial embolization. During perioperative period, nursing was carried out for patients and the patients were explained with the method, purpose, results and cautions about the treatment. Moreover, psychological nursing intervention, dietary intervention, auxiliary examination were performed. The postoperative puncture site and operated limb situations were observed and recorded for medication guidance. In this study, all patients were well-treated. Adverse reactions noted after treatment mainly were liver area pain, digestive tract reaction, fever, which were relatively mild and effectively improved with timely medical measures, with no severe complications. Comparison of quality of life score and mental status score before and after nursing showed significant improvement. To guarantee a smooth and successful implementation of transcatheter arterial embolization for patients with primary liver cancer, the key is to develop a scientific and comprehensive nursing mode to improve curative effect.
\end{abstract}

Key words: Primary liver cancer, transcatheter arterial embolization, comprehensive nursing care

Clinically, primary liver cancer is a common malignant tumor. With the changes in life style, environment and dietary habits, the number of patients with primary liver cancer was on the rise in recent years. Currently, the most common treatment method for such disease is surgical resection. However, as most cases of primary liver cancer are in advanced stage, the recurrence rate after surgery is high and some patients are accompanied with liver cirrhosis, the resection rate of early liver cancer is only $20-30 \%$, while most of cases needed to be treatment with non-surgical scheme ${ }^{[1-3]}$.

At present, information available on the etiology and pathogenesis of primary liver cancer has not reached unified conclusion. It is well-recognized that the primary liver cancer is associated with liver cirrhosis, viral hepatitis, aflatoxin and environment factors. For patients who have missed the best opportunity for 
surgical resection or have no surgery option left, the main treatment approach is to transcatheter arterial embolization, which can prolong the survival time on one hand, and restore opportunity for surgical resection for some patients on the other hand.

During implementation of transcatheter arterial embolization, scientific nursing care is of vital importance. In this study, the nursing effect of primary liver cancer before and after transcatheter arterial embolization was investigated, to provide valuable guidance for practical nursing.

A total of 200 patients with primary liver cancer who were treated in the Second People's Hospital of Huai'an during May 2014 to March 2018 were selected as research subjects. The inclusion criteria were, those who met with the clinical diagnosis and staging criteria of primary liver cancer of Chinese Society of Liver Cancer, Chinese Anticancer Association; those who underwent clinical and imaging examinations such as ultrasonic B, CT, MRI, hepatic arteriography, PET, serum alpha-fetoprotein determination ${ }^{[4,5]}$ and those with no contraindication to surgical treatment. The selected patients enjoyed the right to know and signed informed consent form. Exclusion criteria were, patients accepting other antitumor treatments recently (within one month); patients with severe heart, liver and kidney dysfunction; patients with jaundice, ascites, cachexia and multi-site metastases and patients with poor compliance. Of the 200 selected patients, there were 122 males and 78 females with age ranged from 30 to 75 y (average age 50.6 \pm 3.2 ).

Before treatment, intramuscular injection of $10 \mathrm{mg}$ of diazepam was given as usual for surgical site disinfection and local infiltration anesthesia. Puncture intubation was conducted via arteria cruralis beat area of right groin according to the Seldinger technique. Under the X-ray scan or CT guidance, the tumor site was clearly determined to which the catheter was extended ${ }^{[6]}$. The feeding artery of tumor was found, into which the chemotherapeutic drugs were injected. The interventional drugs used for liver cancer included cisplatin, glycyrrhizin, epirubicin, mitomycin, Brucea javanica oil injection, arsenic trioxide injection, elemene and $40 \%$ iodipin. According to the actual disease condition, 2 or 3 drugs along with iodipin was injected into the artery.

Preoperative nursing method included basic nursing, which was establishing a harmonic patient-doctor relation, helping patients to know ward environment, keeping the ward clean, making patients feel safe and comfortable; dietary nursing involved instructing patients to take semi-liquid diet that was light and easy to digest 1 or $2 \mathrm{~d}$ before surgery, prohibiting patients to drinking water $5 \mathrm{~h}$ prior to surgery. Mental nursing was to establish a sound communication with patients and knowing their mood condition, making targeted measures to eliminate anxiety, tension, panic, depression, informing them about their disease and the purpose, method, effect and cautions to be taken with treatment, so as to remove their fear and improve their confidence and compliance.

Postoperative nursing method included closely focusing patients' disease conditions and monitoring their vital signs such as respiratory rate, blood pressure, pulse, heart rate, body temperature; implementing strict bed rest for $24 \mathrm{~h}$, informing the doctor of abdominal pain or peritoneal irritation that patients might experience. Particular nursing care should be given to puncture site and the affected limb. The postoperative puncture site required bandage for $12 \mathrm{~h}$ and bed rest for $24 \mathrm{~h}$, puncture site bleeding and hematoma should be avoided. Keeping the right leg immobilized for $8 \mathrm{~h}$, examining the skin temperature and color; massaging the lower limb to prevent thrombosis; and instructing patients to drink more water, antipyretics along with cold sponging to be given if the body temperature is over $39^{\circ}$ and observing the gastrointestinal reaction. High doses of chemotherapy drugs exert gastrointestinal toxicity, embolizing agents might enter the peripheral artery of the duodenum, which could cause acute gastrointestinal reactions, thus resulting in nausea and vomiting. Therefore, patients should be instructed to take light diet, if emesis occurs, patient's head should be tilted to one side to prevent aspiration leading to choking or coughing. Finger pressing or acupuncturing was performed at Zusanli or Neiguan to eliminate digestive tract symptoms ${ }^{[7,8]}$. Good nursing care should aim restoring function of liver and kidney. Fluid infusion should be applied regularly before surgery recording urine output and observing urine color after operation, and maintaining urine output around $2500 \mathrm{ml}$.

Rehabilitation guidance was provided which included, postoperative rehabilitation phase in which metal nursing should be strengthened to increase patients' belief in rehabilitation to keep them in positive mood ${ }^{[9]}$. In addition, instructing patients to take appropriate exercise to improve immunity, and imparting patients with health knowledge and rehabilitation information to prevent postoperative complications. Moreover, 
TABLE 1: ADVERSE REACTIONS

\begin{tabular}{lllll}
\hline Number of cases & digestive tract reaction & liver area pain & fever & Total number \\
\hline 200 & 40 & 22 & 25 & 87 \\
\hline
\end{tabular}

TABLE 2: COMPARISON OF QUALITY OF LIFE SCORE BEFORE AND AFTER NURSING

\begin{tabular}{lccccc}
\hline Time & Number of cases & $\begin{array}{c}\text { Physiological } \\
\text { function }\end{array}$ & Mental function & Social function & $\begin{array}{c}\text { Total quality of life } \\
\text { score }\end{array}$ \\
\hline After nursing & 200 & $0.97 \pm 0.32$ & $1.30 \pm 0.52$ & $1.29 \pm 0.15$ & $4.81 \pm 0.55$ \\
Before nursing & 200 & $0.43 \pm 0.29$ & $1.01 \pm 0.05$ & $0.82 \pm 0.23$ & $3.26 \pm 1.20$ \\
t & & 3.58 & 5.31 & 5.09 & 8.36 \\
$\mathrm{P}$ & & $<0.05$ & $<0.05$ & $<0.05$ & $<0.05$ \\
\hline
\end{tabular}

Mean \pm standard deviation

TABLE 3: COMPARISON OF SAS AND SDS SCORE BEFORE AND AFTER NURSING

\begin{tabular}{lccc}
\hline Time & Number of cases & SAS score & SDS score \\
\hline After nursing & 200 & $41.70 \pm 4.25$ & $40.61 \pm 2.80$ \\
Before nursing & 200 & $48.92 \pm 5.05$ & $48.65 \pm 3.86$ \\
$\mathrm{t}$ & & 9.72 & 6.51 \\
$\mathrm{P}$ & & $<0.05$ & $<0.05$ \\
\hline
\end{tabular}

Mean \pm standard deviation

making sure patients take medication, diet, rest and reexamination strictly as prescribed ${ }^{[10-15]}$.

The adverse reactions were recorded. The mental status (anxiety scores, depression scores) and quality of life before and after nursing were compared. SPSS21.0 software was used for statistical analysis. The data was expressed as mean \pm standard deviation and intergroup differences were compared using $t$ test. The enumeration data was expressed as natural number (n), percent (\%), and the intergroup difference was tested by $\chi^{2}$. The difference was of statistical significance when $p<0.05$.

As shown in Table 1, the adverse reactions observed mainly included liver area pain, gastrointestinal reaction, fever, all of which were relatively mild and were improved with timely measures, without causing severe complications such as puncture site bleeding, hematoma, deep vein thrombosis. As shown in Table 2 the quality of life scores after nursing intervention are significantly higher than those before nursing $(\mathrm{p}<0.05)$. As shown in Table 3, the self-rating anxiety scale and self-rating depression scale scores after nursing are higher than those before nursing, $\mathrm{p}<0.05$.

Early detection and early treatment is the key influencing the curative effect of primary liver cancer. Currently, the 5-year survival rate of primary liver cancer after surgical treatment is $70 \%{ }^{[16]}$, which is relatively satisfactory. So patients should not lose confidence and accept treatment as soon as possible if diagnosed with primary liver cancer.

One of the main measures of treating advanced primary liver cancer is transcatheter arterial embolization.
Drugs are injected into tumor side of liver via catheter to increase the concentration of chemotherapeutic drugs in the blood perfusing tumor region and exert good cytotoxic effect on tumor cells. In addition, adverse reaction caused by systemic chemotherapy could be prevented. Scientifically sound nursing intervention is the key to guarantee smooth implementation of transcatheter arterial embolization. Therefore, the preoperative preparation work should be improved and real-time monitoring of vital signs of patients should be implemented to prevent emergency situations. Standard nursing operation should be carried out to prevent complications, increase patients' quality of life and accelerate rehabilitation. In conclusion, to guarantee smooth implementation of transcatheter arterial embolization for patients with primary liver cancer, scientific and comprehensive nursing mode should be developed to improve curative effect. This nursing mode can prolong patients' survival time improve their quality of life, which has great clinical application.

\section{REFERENCES}

1. Zhang LY, Wang YZ. Observation of nursing effect before and after transcatheter arterial embolization for patients with primary liver cancer. J Tradit Chin Med 2018;34(01):110-111.

2. Li H, Lu Q, Yang P, Liu WR, Wang HY, Li SM. Symptoms of patients accepting primary liver cancer surgery and their needs for continuing care. Chin J Nursing 2015;50(06):684-88.

3. Ma HL. Related factors of causing acute upper gastrointestinal hemorrhage after transcatheter arterial embolization for patients with primary liver cancer and nursing mode. World Med Inform 2015;15(43):69-70.

4. Nouso K, Miyahara K, Uchida D. Effect of hepatic arterial infusion chemotherapy of 5-fluorouracil and cisplatin for advanced hepatocellular carcinoma in the nationwide survey of 
primary liver cancer in Japan. Br J Cancer 2016;109(7):19047.

5. Asayama Y, Yoshimitsu K, Irie H. Poorly versus moderately differentiated hepatocellular carcinoma: vascularity assessment by computed tomographic hepatic angiography in correlation with histologically counted number of unpaired arteries. J Comput Assist Tomogr 2017;31(2):188-92.

6. Han YH. Analysis of reasons of fever occurring after implementing transcatheter arterial chemo-embolization for patients with primary liver cancer and relevent nursing mode analysis. World Med Inform 2016;16 (64):288-91.

7. Jin X, Fang F, Yu JX. Research progress on nursing for vomiting after implementing transcatheter arterial chemoembolization for patients with primary liver cancer. Shanghai Nurs J 2017;17(06):62-5.

8. Zhang Y, Zhao J, Guo D, Zhong W, Ran L. Evaluation of short-term response of high intensity focused ultrasound ablation for primary hepatic carcinoma: utility of contrastenhanced MRI and diffusion-weighted imaging. Eur J Radiol, 2016;79(3):347-52.

9. Gong L, Shi L, Sun J, Yuan WS, Chen JF, Liu P, et al. Comparative survival analysis of adjuvant therapy with iodine-131-labeled lipiodol to hepatic resection of primary hepatocellular carcinoma: A meta-analysis. Nucl Med Commun 2017;35(5):484-92.

10. Gao W, Wang Y, Basavanagoud B, Jamil MK. Characteristics Studies of Molecular Structures in Drugs. Saudi Pharm J 2017;25(4):580-6.

11. Ge S, Wang L, Liu Z, Jiang S, Yang X, Yang W, et al. Properties of Nonvolatile and Antibacterial Bioboard Produced From Bamboo Macromolecules by Hot Pressing. Saudi J Biol Sci 2018;25(3):474-78.
12. Alkowni R, Jodeh S, Hussein F, Jaradat N. Phytochemical Screening and Antibacterial Activity of Cyclamen Persicum Mill Tuber Extracts. Pak J Pharm Sci 2018;31(1):187-92.

13. Al-Enazi NM, Awaad AS, Zain ME, Alqasoumi SI. Antimicrobial, Antioxidant and Anticancer Activities of Laurencia catarinensis, Laurencia majuscula and Padina pavonica Extracts. Saudi Pharm J 2018;26(1):44-52.

14. Afsar CU, Karabulut M, Karabulut S, Alis H, Binboga $\mathrm{S}$, Bilgin $\mathrm{E}$, et al. Circulating Monocyte Chemoattractant Protein-1 (Mcp-1/Ccl-2) as a Potential Biomarker for the Diagnosis of Pancreatic Adenocarcinoma. Acta Medica Mediterr 2017;33(3):501-7.

15. Ai F, Wang J, Li Y, Ma Y. Effect of Drug Particle Size On Complexation, Physicochemical Properties and Dissolution of Cyclodextrin Inclusion Complexes. Indian J Pharm Sci 2017;79(1):131-8.

16. Dong X, Jin K, Hu X. Antitumor effect of FP3 in combination with cetuximab on patient-derived tumor tissue xenograft models of primary colon carcinoma and related lymphatic and hepatic metastases. Int J Mol Med 2016;30(1):126-32.

This is an open access article distributed under the terms of the Creative Commons Attribution-NonCommercial-ShareAlike 3.0 License, which allows others to remix, tweak, and build upon the work non-commercially, as long as the author is credited and the new creations are licensed under the identical terms

This article was originally published in a special issue: Special issue on "Drug Development and Human Health in China"

Indian J Pharm Sci 2020:82(1)spl issue2;48-51 